Direct deposition of single-walled carbon nanotube thin films via electrostatic spray assisted chemical vapor deposition

This article has been downloaded from IOPscience. Please scroll down to see the full text article.

2009 Nanotechnology 20065601

(http://iopscience.iop.org/0957-4484/20/6/065601)

The Table of Contents and more related content is available

Download details:

IP Address: 140.112.113.225

The article was downloaded on 26/06/2009 at 11:45

Please note that terms and conditions apply. 


\title{
Direct deposition of single-walled carbon nanotube thin films via electrostatic spray assisted chemical vapor deposition
}

\author{
Ya-Ping Hsieh ${ }^{1,2}$, Mario Hofmann ${ }^{1}$, Hyungbin Son ${ }^{1}$, Xiaoting Jia ${ }^{3}$, \\ Yang-Fang Chen ${ }^{2}$, Chi-Te Liang ${ }^{2}$, Mildred S Dresselhaus ${ }^{1,4}$ and \\ Jing Kong ${ }^{1}$ \\ ${ }^{1}$ Department of Electrical Engineering and Computer Science, Massachusetts Institute of \\ Technology, Cambridge, MA 02139-4037, USA \\ ${ }^{2}$ Department of Physics, National Taiwan University, Taipei 106, Taiwan \\ ${ }^{3}$ Department of Material Science and Engineering, Massachusetts Institute of Technology, \\ Cambridge, MA 02139-4037, USA \\ ${ }^{4}$ Department of Physics, Massachusetts Institute of Technology, Cambridge, \\ MA 02139-4037, USA, \\ E-mail: ctliang@phys.ntu.edu.tw and jingkong@mit.edu
}

Received 15 October 2008, in final form 21 November 2008

Published 15 January 2009

Online at stacks.iop.org/Nano/20/065601

\begin{abstract}
In this work, electrostatic spray assisted chemical vapor deposition is used to directly deposit single-walled carbon nanotube (SWNT) thin films on a substrate. The catalyst solution was finely dispersed by a strong electrical field and injected into the heated reaction zone $\left(950-1100^{\circ} \mathrm{C}\right)$ during the growth. It was found that under optimized growth conditions, the deposited material consists of SWNTs while only small amounts of impurities are observed. The growth at different temperatures results in nanotubes of different length and morphology. The location at which the SWNTs deposit at the downstream end of the growth chamber is found to be affected by the nanotube length and the growth temperature, which can be understood by considering different forces acting on the floating aerosol particles inside the furnace. These results suggest a potential for in situ separation of the SWNTs by applying different forces to the floating SWNTs.
\end{abstract}

(Some figures in this article are in colour only in the electronic version)

\section{Introduction}

Single-walled carbon nanotube (SWNT)-based monolayer- or submonolayer thin films on substrates represent a material platform that is attractive for many applications, such as thin film transistors for display and flexible electronics, transparent electrodes [1-6], substrates for neuron cell growth and stimulation [7], etc. In order to obtain such a thin film of SWNTs on substrates, most approaches are solution-based, i.e., a bulk amount of SWNT material is purified and dispersed in solution, and is deposited on the substrate afterward via different techniques, such as drop casting, spraying [5, 6] or vacuum filtration [4]. The purification and dispersion steps are costly and time consuming. More importantly, due to the sonication treatment during these steps, defects are created along the length of SWNTs, and the nanotubes are cut to an average length of $1 \mu \mathrm{m}$ or less. As a result, the electrical properties of these SWNTs films are degraded. Here we use a floating catalyst chemical vapor deposition (CVD) method to directly coat a substrate with SWNT thin films. The catalyst is dispersed into an aerosol produced by electrospraying since this method $[8,9]$ is expected to provide small, highly charged droplets with a narrow diameter distribution when using a strong applied electrical field [10]. These droplets of ethanol solution containing ferrocene provide both the catalyst and carbon precursor for the SWNT growth. Once the nanotubes are generated in the hot zone, they are carried by the gas flow and deposit on a low temperature surface at 


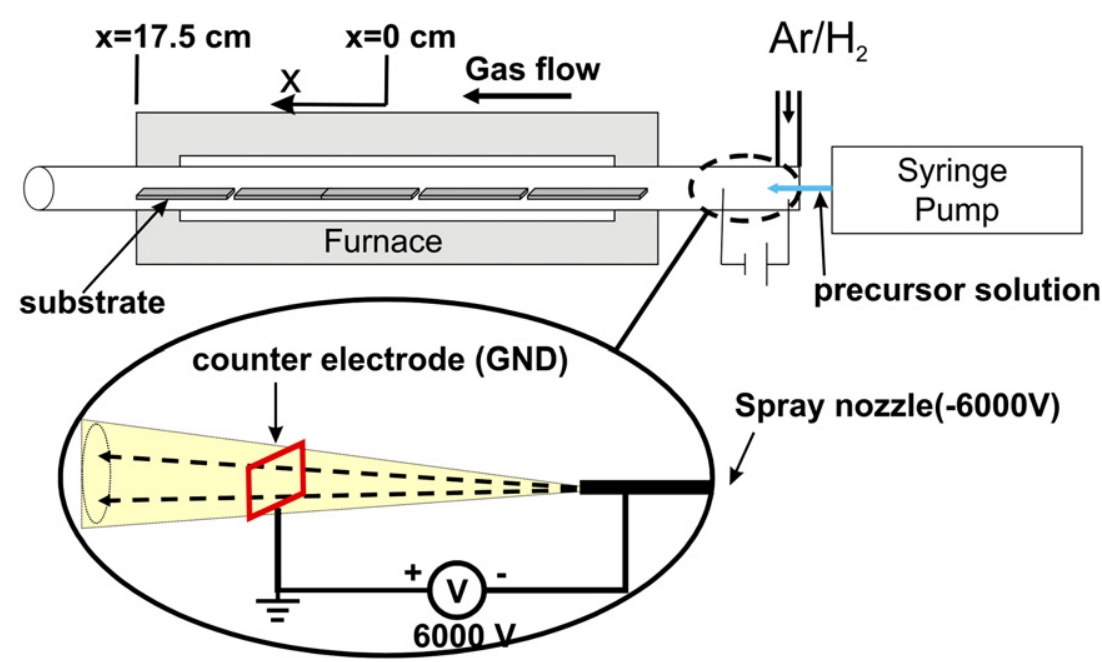

Figure 1. A schematic diagram of the synthesis setup: $x$ is the position along the CVD chamber, and $x=0$ is designated to be the furnace center. The edge of the furnace at the downstream end is $x=17.5 \mathrm{~cm}$. Inset: close up of the spray nozzle with an indication of the electrical field.

the downstream end of the furnace. Atomic force microscopy (AFM) and Raman spectroscopy are used as characterization techniques to examine where the nanotubes deposit inside the furnace. It was found that the SWNTs are mainly deposited at specific locations, instead of uniformly coating the inside of the reactor. By considering different types of forces acting on the floating SWNTs, the preferential SWNT deposition at specific locations can be understood. By optimizing the CVD process parameters, films consisting of mostly isolated SWNTs could be obtained, as confirmed by comparison of Raman and AFM size distributions. Furthermore, an investigation of the length distributions of nanotubes deposited at different locations indicates the potential of in situ SWNT sorting.

\section{Experimental details}

Figure 1 is a schematic depiction of our synthesis setup: ethanol (99.9\%, Aldrich) with $0.01 \mathrm{M}$ Ferrocene (99\%, Aldrich, $1.86 \mathrm{mg} \mathrm{ml}^{-1}$ ) is used as the catalyst and carbonaceous precursor, respectively, and is introduced into a spray nozzle. The solution is dispersed into an aerosol when the surface tension of the liquid/air interface is overcome by a strong electrostatic field (on the order of $10^{6} \mathrm{~V} \mathrm{~m}^{-1}$ ) applied between the spray nozzle $(-6 \mathrm{kV})$ and the counter electrode (ground) (as indicated in the inset of figure 1). Subsequently small droplets are extracted from the spray nozzle. The precursor solution is delivered to the spray nozzle at a flow rate of $8 \mathrm{ml} \mathrm{h}^{-1}$. The atomized and charged droplets of the precursor solution are carried into the growth chamber by a flow of argon/hydrogen (with a volume ratio of 61/39 and a total flow rate of $1000 \mathrm{sccm})$. Silicon substrates with $100 \mathrm{~nm}$ thermally grown oxide $\left(\mathrm{SiO}_{2} / \mathrm{Si}\right)$ were placed inside the chamber to collect the grown nanotubes (figure 1). In order to investigate the deposition of SWNTs throughout the furnace, long $\mathrm{SiO}_{2} / \mathrm{Si}$ substrates were stacked to cover the entire length of the furnace $(35 \mathrm{~cm})$. We chose the center of the furnace to be the origin $(x=0)$ of our distance measurement. Three growth temperatures, 950, 1000 and $1100^{\circ} \mathrm{C}$, were used in this study. The growth time was usually $4 \mathrm{~min}$, after which the catalyst delivery was turned off and the samples were cooled down under $\mathrm{Ar} / \mathrm{H}_{2}$ flow. After the CVD process, AFM imaging and confocal Raman mapping across the surface of the substrates were used to characterize the SWNT deposition. The temperature inside the furnace and substrate temperatures at different locations were measured using two K-type thermocouples that reached into the reaction zone.

\section{Results and discussion}

In order to characterize the materials deposited on the substrates, confocal Raman spectroscopy (with a laser spot of $\sim 1 \mu \mathrm{m}^{2}$ ) was used to scan a line across the substrates, with a step size of $35 \mu \mathrm{m}$ using an excitation wavelength of $532 \mathrm{~nm}$. The $\mathrm{G}$ band, a Raman mode at around $1600 \mathrm{~cm}^{-1}$ and associated with the in-plane vibrations of $\mathrm{sp}^{2}$ carbonaceous species was used to examine the deposition of the obtained material. The intensity variation of this Raman feature across the sample can give an overview of the spatial distribution of the nanotube density [11]. Figure 2(a) presents the integrated $\mathrm{G}$ band intensity $\left(1500-1650 \mathrm{~cm}^{-1}\right)$ versus position $x$ (black) for three different growth temperatures. It can be seen that most carbonaceous material is deposited in the region close to the downstream end of the furnace ( $x=17.5 \mathrm{~cm}$, figure 1). In other locations, almost no $\mathrm{G}$ band intensity is observed.

The integrated intensity of the Raman D band around $1300 \mathrm{~cm}^{-1}$ (from 1250 to $1400 \mathrm{~cm}^{-1}$ ) versus position $x$ for these three growth temperatures are also shown in figure 2(a) underneath the corresponding $\mathrm{G}$ band distribution. The $\mathrm{D}$ band in the Raman spectrum is known to arise from the defects in $\mathrm{sp}^{2}$ structures. The average ratio of our $\mathrm{G} / \mathrm{D}$ band intensities is about 50. This low D band intensity indicates that our samples contain high quality graphitic structures and a low content of amorphous carbon material. This is consistent with figures 2(b)-(d) where AFM images were taken at the center of the density distributions for $950{ }^{\circ} \mathrm{C}$ (b), $1000^{\circ} \mathrm{C}$ (c), 

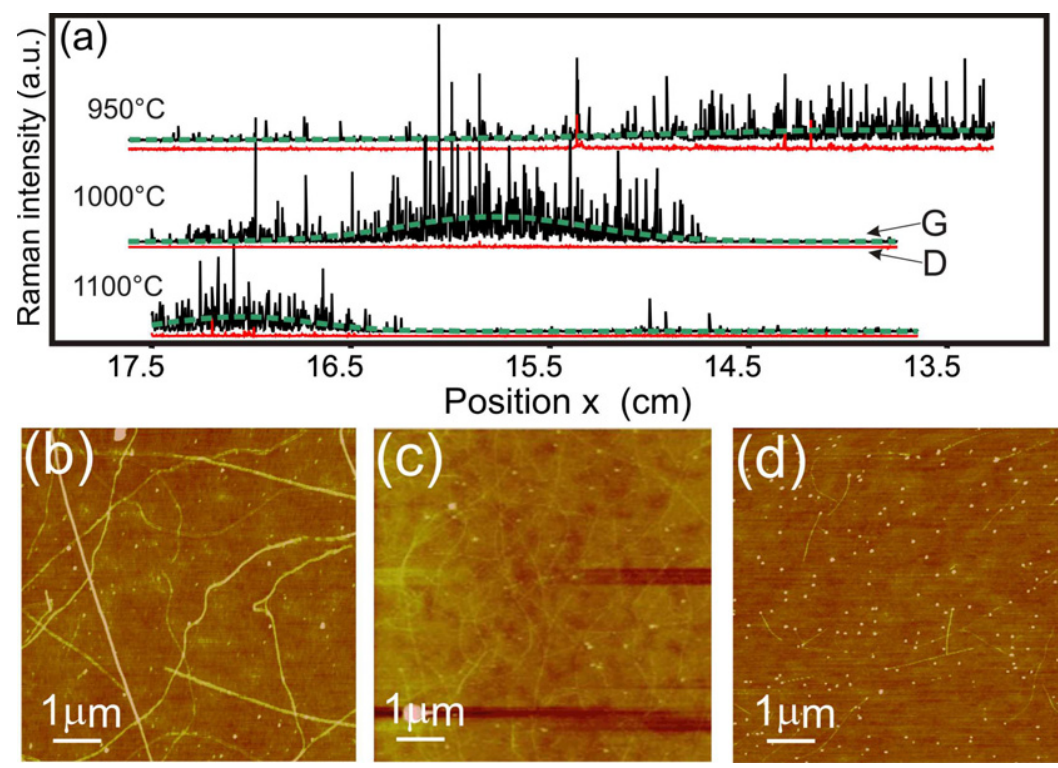

Figure 2. (a) Spatial distribution of the integrated G band intensity $\left(1500-1650 \mathrm{~cm}^{-1}\right)$ (black), the integrated D band intensity $\left(1250-1400 \mathrm{~cm}^{-1}\right)$ (red) and the fitted G band intensity (green, dashed) for three different growth temperatures: 950,1000 and $1100{ }^{\circ} \mathrm{C}$. (b) -(d) Representative AFM images of the sample morphology taken at the center of the density distributions for $950{ }^{\circ} \mathrm{C}(\mathrm{b}), 1000{ }^{\circ} \mathrm{C}(\mathrm{c})$, and $1100^{\circ} \mathrm{C}(\mathrm{d})$ growth temperatures.
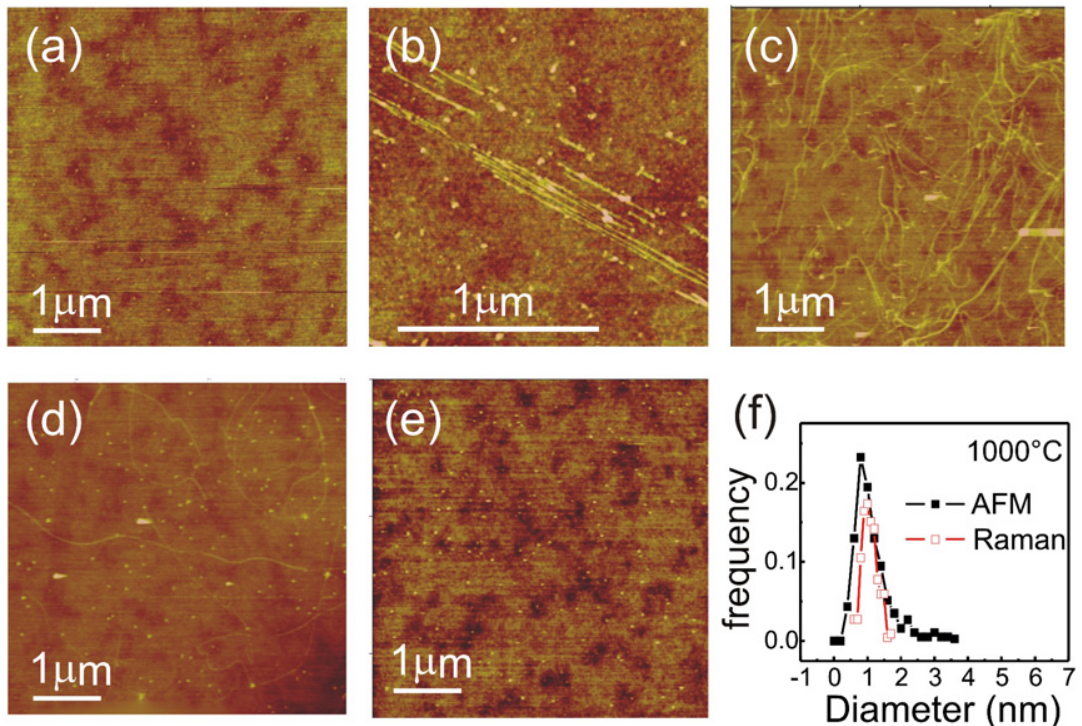

Figure 3. Representative AFM images of the growth results at different positions $x$ along the furnace: (a) $14 \mathrm{~cm}$, (b) $14.5 \mathrm{~cm}$, (c) $15.5 \mathrm{~cm}$, (d) $16.5 \mathrm{~cm}$ and (e) $17.5 \mathrm{~cm}$ (growth temperature $1000^{\circ} \mathrm{C}$ ). (f) Diameter distribution of the SWNTs around position (d) as obtained by Raman and AFM measurements taken from 435 and 450 nanotubes, respectively.

and $1100{ }^{\circ} \mathrm{C}$ (d) growth temperatures and they show that most of the deposited structures are SWNTs. The average spatial distribution of the $\mathrm{G}$ band intensities (green curves in figure 2(a)) follows a Gaussian distribution and its center shifts toward the downstream edge as the growth temperature increases. On the other hand, the width of the fitted Gaussian distribution becomes larger at lower growth temperatures. From the $\mathrm{G}$ band intensity distribution in figure 2(a) it can be seen that in order to obtain a uniform coating on the substrate, the sample size would have to be limited to $2 \mathrm{~cm}$ for the $1000^{\circ} \mathrm{C}$ case, whereas for the $950{ }^{\circ} \mathrm{C}$ growth, there is a wider range $(>3 \mathrm{~cm})$ for an even coat of SWNTs on the substrate.
Figure 3 presents a series of AFM images taken on substrates at different locations inside the furnace for the growth temperature of $1000^{\circ} \mathrm{C}$. The density of SWNTs in figures $3(\mathrm{a})-(\mathrm{e})$ is consistent with the measured variation of the average integrated $\mathrm{G}$ band intensity in figure 2(a).

The diameter distribution of the nanotubes is derived independently from analysis of AFM images from 450 nanotubes and their Raman characterization (figure 3(f)). Confocal Raman spectra with eight different laser excitation wavelengths $(457,488,514,532,594,647,675,780 \mathrm{~nm})$ were taken in a $200 \mu \mathrm{m} \times 40 \mu \mathrm{m}$ area in the same spatial region as the AFM images were taken to ensure a representative 
coverage of nanotubes with different resonance condition. The diameter of each individual nanotube was determined from the frequency of its RBM mode $[12,13]$ using formula $A=$ $218.8 / B-15.9$ where $A$ and $B$ are the frequency of the RBM mode and the diameter, respectively. Good agreement between the two diameter distributions derived by AFM and Raman measurements (figure 3(f)) is observed. This indicates the absence of a significant amount of nanotube bundles because the diameter derived from the AFM height measurementrepresenting the diameter of the entire bundle-would differ from the distribution from Raman analysis which only probes the diameters of individual SWNTs within this bundle. Thus it can be seen that for the growth at $1000^{\circ} \mathrm{C}$ most deposited nanotubes are isolated SWNTs, which is very important for certain applications $[14,15]$. We attribute this high probability of depositing isolated SWNTs to the fact that we used a much more dilute concentration of both the ferrocene catalyst and the carbon precursor (compared with [16], the carbon precursor is about 16 times more dilute in the carrier gas and the ferrocene to carbon precursor ratio is about ten times less) as well as the small diameter of the generated aerosol particles that is expected from electro-spray dispersion. As a result, the asgrown SWNT aerosol concentration inside the furnace is much reduced and the nanotubes have less chance to interact with each other in the gas phase to form bundles. Furthermore it is worth mentioning that the diameter distribution is fairly narrow, $\sim \pm 0.3 \mathrm{~nm}$, and the average diameter is $<1 \mathrm{~nm}$, which makes these nanotubes very useful for optical and spectroscopic applications [17, 18].

In order to understand the distribution of SWNT density as a function of their position on the substrate, thermocouple temperature measurements were conducted across the cross section of the reaction chamber (termed radial direction as the chamber is cylindrical) and along the length of the reaction chamber (termed $x$-direction in figure 1). Figure 4 displays the gas-temperature (figure 4(a)) and the temperature gradients $\Delta T$ in the radial (figure 4(b)) and $x$-direction (figure 4(c)) as a function of position $x$ along the chamber. The radial temperature gradient is approximated by taking the difference of the gas and the substrate temperature at the same $x$. It is expected that the walls (which are subjected to a more intimate heat transfer from the furnace and the environment) are cooling down faster outside the heating zone of the furnace than the gas stream, and indeed it can be seen in figure 4(c) that $\Delta T$ in the radial direction increases in magnitude towards the downstream edge of the furnace. This temperature gradient will give rise to a thermophoretic force on the floating SWNTs in the radial direction [19], driving them towards the colder substrate and depositing them on the substrate. In addition, the floating aerosol particles (including SWNTs) in the gas stream experience other types of driving forces that will move them in the $x$-direction: (1) diffusion because of a density gradient of the produced SWNTs along the furnace will direct them from the reaction zone, where they are grown, towards the two ends of the furnace tube; (2) a thermophoretic force in the $x$-direction due to the changes in temperature along the $x$ direction as shown in figure 4(a) will give rise to a motion of the particle away from the hot reaction zone; (3) the gas flow

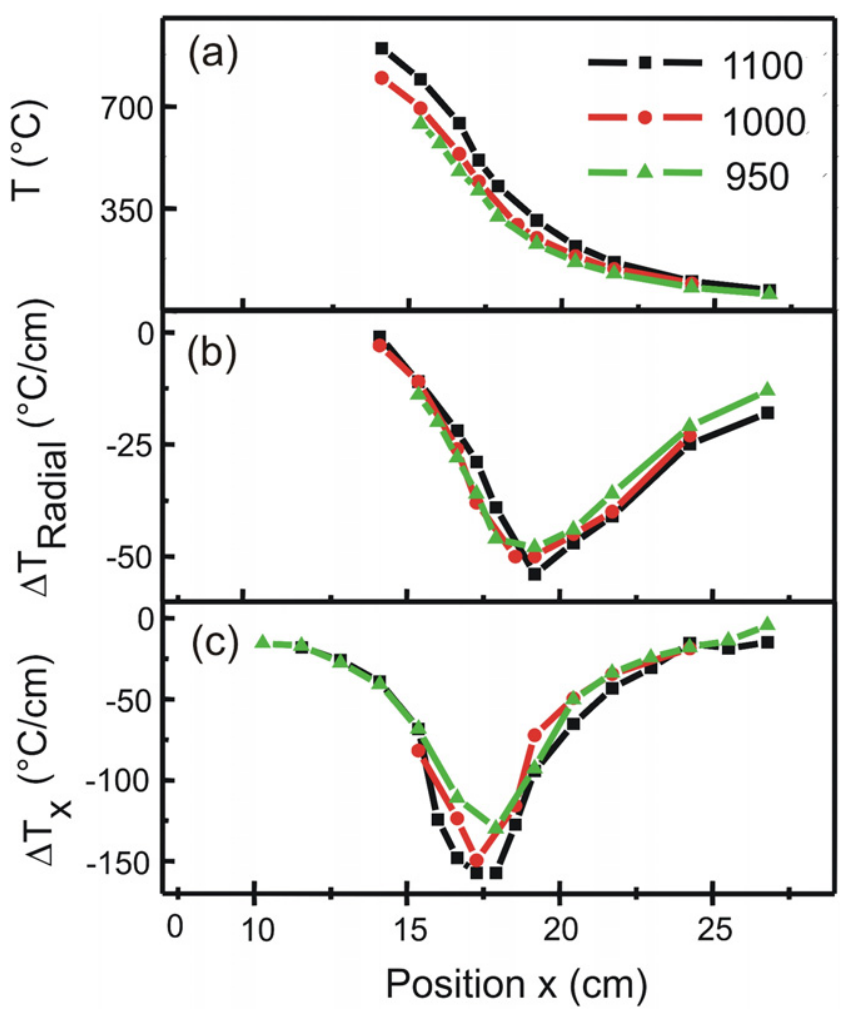

Figure 4. (a) Spatial temperature distribution along the $x$ direction. Spatial temperature gradient distribution (b) in the radial and (c) $x$ directions.

will exert a drag force on the particle towards the downstream direction. This drag force is strongly dependent on the size of the particles, especially if they become comparable to the mean free path of the gas molecules [20]. From figure 3(b) we can see that as we follow the gas flow direction from upstream to downstream, the materials which precipitated on the substrate first consists of very short, broken pieces of SWNTs. We attribute this to the fact that since these pieces are short, the drag force on them due to the gas flow is small. At the same time the small mass of these particles gives them larger mobility in small temperature gradients, which appear closer to the reaction zone. As a result these fragments start to precipitate at an earlier location than larger nanotubes.

Longer nanotubes, on the other hand experience (apart from the diffusion and thermophoretic forces) a stronger drag force and are carried further downstream and deposit at a later position. This implies that the floating catalyst CVD can be potentially used for in situ separation of SWNTs if varying forces can be applied to different types of SWNTs. Thus it is possible for SWNTs of different lengths to be spatially separated. Indeed, the results in figure 5 confirm this point. Figures 5(a) and (c) are AFM images of SWNTs grown at $1100{ }^{\circ} \mathrm{C}$ and collected at $16.2 \mathrm{~cm}$ and $17.4 \mathrm{~cm}$ respectively. (Growth conditions correspond to the ones used in figure 2(a).) A high ethanol decomposition rate at this temperature results in carbon coating over the catalyst (as indicated by the increased size of catalyst clusters in figures 5(a) and (c)) and therefore the catalyst lifetime becomes shorter. As a result, the SWNTs are much shorter compared to the 950 and $1000^{\circ} \mathrm{C}$ cases. These 

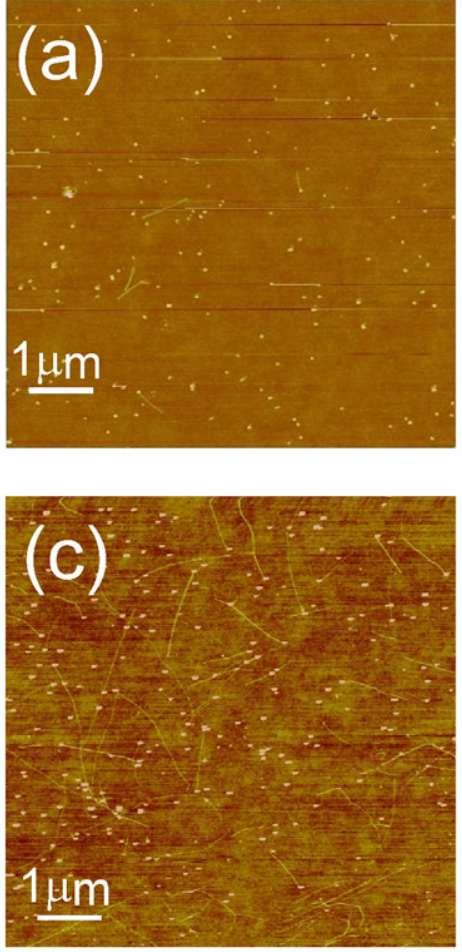
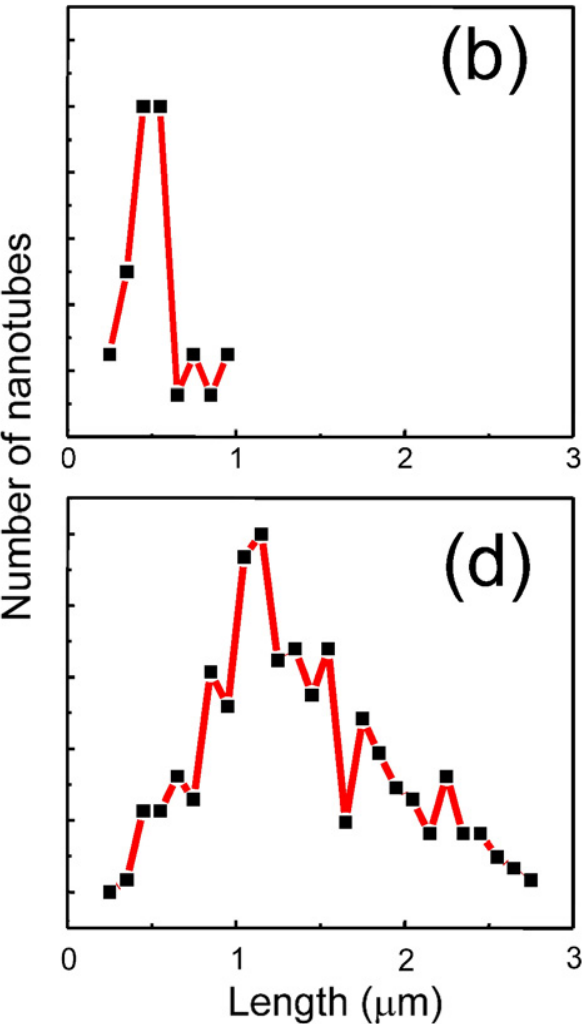

Figure 5. AFM images of SWNTs grown at $1100^{\circ} \mathrm{C}$ and collected at $x=16.2 \mathrm{~cm}$ (a) and $17.4 \mathrm{~cm}$ (c) respectively. Length distributions for the obtained nanotubes at $x=16.2 \mathrm{~cm}(\mathrm{~b})$ and $17.4 \mathrm{~cm}(\mathrm{~d})$. Growth temperature was $1100^{\circ} \mathrm{C}$.

short SWNTs simplify the observation of the length dependent separation by AFM. Figures 5(b) and (d) show the length distributions of the SWNTs collected at the same positions (28 SWNTs and 288 SWNTs are used for the distributions, respectively). It can be seen that SWNTs with an average length $<1 \mu \mathrm{m}$ precipitate earlier than SWNTs with average length $>1 \mu \mathrm{m}$.

Our analysis of the various forces acting on the floating aerosol particles (SWNTs and catalyst particles, etc) helps to explain other experimental observations as well. For example, if the growth conditions are not tuned well (i.e. when using too high concentrations of carbon precursor), amorphous carbon will deposit even at the upstream edge of the furnace (region -16.5 to $-17.5 \mathrm{~cm}$ ). This can be understood if one considers the following scenario: the floating amorphous carbon particles are too small to experience a significant drag force from the gas flow. Since there are diffusive and thermophoretic forces in both directions along the chamber they can drive the particles from the hot zone to both ends of the furnace. As a result, some of these particles are moving against the flow and are finally deposited at the upstream cold region.

Finally we note that the comparison of the SWNT spatial distributions of the three growth temperatures provides further insights into the growth of the SWNTs. As shown in figure 2(a), the center of the average spatial distribution shifts toward the downstream edge as the growth temperature increases. On the other hand, figures 4(b) and (c) show that both the axial and the radial temperature gradients are very similar in the regions of interest for the three growth temperatures (figure 4(a)) and cannot explain the observed trends. However, the diffusion of nanotubes away from the reaction zone is expected to increase with increasing temperature and indeed we see that nanotubes grown at $1100^{\circ} \mathrm{C}$ deposit further downstream than the ones grown at 1000 or $950^{\circ} \mathrm{C}$. This result also sheds some light on the lesser importance of drag force compared to diffusivity: since the average length of the nanotubes grown at $950^{\circ} \mathrm{C}$ is much higher than the nanotubes grown at $1100^{\circ} \mathrm{C}$, those long nanotubes would experience larger drag force and deposit further downstream, which would cause the opposite trend to the increase in diffusion explained before. Instead the temperature and inverse length dependences of the diffusivity [21] prevail and cause the observed tendency in the center position of the density distribution.

\section{Conclusions}

In this work, we used floating catalyst CVD to directly deposit very thin films of SWNTs on substrates, which is important for many applications. If a low nanotube concentration condition (i.e., low catalyst concentration and low ethanol injecting rate) is maintained, an isolated nanotube film can be obtained. The deposition of floating SWNTs on the substrate can be explained by analyzing the forces acting on them. A length separation of nanotubes deposited at different positions was observed which shows potential for future applications. 


\section{Acknowledgments}

This work was partially funded by the Materials, Structure and Device center, one of the five centers in the Focus Center Research Program (FCRP). YPH acknowledges financial support from the National Science Council of the Republic of China through Project No. 096-2917-I-002-005.

\section{References}

[1] Snow E S, Campbell P M, Ancona M G and Novak J P 2005 Appl. Phys. Lett. 86033105

[2] Takenobua T, Takahashi T, Kanbara T, Sukagoshi K T, Aoyagi Y and Iwasa Y 2006 Appl. Phys. Lett. 88033511

[3] Zhang M, Fang S, Zakhidov A A, Lee S B, Aliev A E, Williams C D, Atkinson K R and Baughman R H 2006 Science 3091215

[4] Wu Z et al 2004 Science 3051273

[5] Duggal R, Hussain F and Pasquali M 2006 Adv. Mater. 1829

[6] Li Y, Kinloch I A and Windle A H 2004 Science 304276

[7] Mazzatenta A, Giugliano M, Campidelli S, Gambazzi L, Businaro L, Markram H, Prato M and Ballerini L 2007 J. Neurosci. 276931

[8] Wei M, Zhi D and Choy K 2006 Nanotechnology 17181
[9] Li W Z, Wen J G and Ren Z F 2002 Appl. Phys. A 74397

[10] Chen D, Pui D Y H and Kaufmar S L 1995 J. Aerosol Sci. 26963

[11] Hart A J, Slocum A H and Royer L 2006 Carbon 44348

[12] Araujo P T, Doorn S K, Kilina S, Tretiak S, Einarsson E, Maruyama S, Chacham H, Pimenta M A and Jorio A 2007 Phys. Rev. Lett. 98067401

[13] Rao A M et al 1997 Science 275187

[14] Furtado C A, Kim U J, Gutierrez H R, Pan L, Dickey E C and Eklund P C 2004 J. Am. Chem. Soc. 1266095

[15] Wang H, Zhou W, Ho D L, Winey K I, Fischer J E, Glinka C J and Hobbie E K 2004 Nano Lett. 41789

[16] Zhu H W, Xu C L, Wu D H, Wei B Q, Vajtai R and Ajayan P M 2002 Science 296884

[17] Yamashita S, Inoue Y, Maruyama S, Murakami Y, Yaguchi H, Jablonski M and Set S Y 2004 Opt. Lett. 291581

[18] Schibli T R, Minoshima K, Kataura H, Itoga E, Minami N, Kazaoui S, Miyashita K, Tokumoto M and Sakakibara Y 2005 Opt. Express 138025

[19] Batchelor G K and Shen C 1985 J. Colloid Interface Sci. 10721

[20] Matthews M T and Hill J M 2006 Q. J. Mech. Appl. Math. 59191

[21] Tirado M M, Martinez C L and Delatorre J G 1984 J. Chem. Phys. 812047 